\section{$\mathbf{m} / \mathbf{S}$}

médecine/sciences $1993 ; 9: 373-5$

\title{
LES GÉNODERMATOSES PIGMENTAIRES : UNE HISTOIRE DE SOURIS ET D'HOMMES
}

\section{Jean-Paul Ortonne}

\footnotetext{
ADRESSE

J.-P. Ortonne : praticien hospitalier, professeur des universités. Service de dermatologie, centre hospitalier universitaire de Nice, hôpital Pasteur, 30, avenue de la Voie-Romaine, BP 69, 06002 Nice Cedex 1, France.

a couleur de la peau et des poils résulte de la présence de mélanines, un groupe hétérogène de pigments comprenant les eumélanines et les phaeomélanines associées respectivement aux couleurs noire ou marron, et aux couleurs jaune-rouge. Ces mélanines sont produites par des cellules très spécialisées, originaires de la crête neurale (voir l'article de C. Dulac, p. 417 de ce numéro), les mélanocytes, qui possèdent une enzyme, la tyrosinase, jouant un rôle clé dans la biosynthèse de ces pigments (voir l'article de B. Bouchard, p. 425 de ce numéro). Les mélanines sont synthétisées dans des organites spécifiques, les mélanosomes, qui sont ultérieurement transférés aux kératinocytes épidermiques via les dendrites mélanocytaires (voir l'article de J.-M. Naeyaert et J.-Ph. Lacour, p. 431 de ce numéro). L'altération de ces processus détermine la survenue de troubles de la pigmentation cutanéophanérienne dont un grand nombre ont un support génétique. Certaines de ces génodermatoses pigmentaires ont perdu une partie de leur mystère, grâce au clonage et au séquençage des gènes touchés par des mutations associées, chez la souris, à des anomalies de la couleur du pelage.

En effet, les gènes murins dits de "pigmentation " ont été particulièrement bien étudiés. Plus de 50 loci ont été identifiés, et regroupés par catégories selon qu'ils contrôlent : (1) la migration, la prolifération et la survie des mélanoblastes et des mélanocytes ; (2) la quantité de mélanines produite ; (3) le type biochimique des mélanines ; (4) la morphologie et la structure des mélanocytes et des mélanosomes. Dans le premier groupe, l'identification de gènes contrôlant l'embryogenèse du système mélanocytaire, notamment les gènes de certains récepteurs à activité tyrosine kinase (TK) $(\mathrm{m} / \mathrm{s}$ $n^{\circ} 10$, vol. $8, p .1106$ ) ou de gènes de développement, a fait progresser significativement notre compréhension du déterminisme de " neurocristopathies pigmentaires " comme le piébaldisme et le syndrome de Waardenburg. Ces génodermatoses se caractérisent cliniquement par une hypomélanose cutanéophanérienne associant une mèche blanche frontale, des macules cutanées achromiques de l'abdomen et des parties moyennes des membres, liées à une absence de mélanocytes. Si, dans la majorité des cas, le piébaldisme est une affection purement dermatologique, le syndrome de Waardenburg comporte des manifestations extra-cutanées, suggérant l'atteinte de plusieurs compartiments de l'" organe mélanocytaire " et de plusieurs dérivés de la crête neurale. Le système proto-oncogène $c$-kit/gène Steel, codant pour le récepteur à activité tyrosine kinase du facteur de croissance mastocytaire (MGF), joue un rôle fondamental dans l'embryogenèse du système mélanocytaire et dans l'homéostasie post-natale du pool mélanocytaire des follicules pileux de la souris. La mise en évidence de mutations de ces gènes chez des souris atteintes de piébaldisme (white spotting) a été rapidement suivie par la détection 
de mutations d'un gène correspondant chez l'homme. Il a été ainsi établi que l'hétérogénéité des phénotypes cliniques du piébaldisme humain correspond à une hétérogénéité génétique, résultant de la diversité des mutations qui affectent le gène $c$ kit [1], mais également de l'implication d'autres gènes. La mutation murine Patch associée à un phénotype pie résulte d'une délétion du gène codant pour la sous-unité $\alpha$ du récepteur du PDGF. Une délétion de ces deux gènes ( $P D G F-R \alpha$ et $c$-kit) a été observée chez un patient atteint du piébaldisme [2].

La mutation Splotch de la souris induit un phénotype proche de celui du syndrome de Waardenburg. Le locus Splotch code pour le gène Pax-3 qui est exprimé dans le tube neural dorsal pendant la deuxième semaine de gestation. Des mutations de $P A X-3$ (HuP 2) ont été détectées chez des patients atteints de ce syndrome. L'hétérogénéité clinique du syndrome de Waardenburg paraît résulter de la diversité des mutations qui atteignent $P A X-3$ [3] et l'implication d'un autre gène dans cette affection n'a pas encore été démontrée.

En ce qui concerne les gènes de pigmentation contrôlant la mélanogenèse, et en particulier la quantité de mélanines synthétisées par les mélanocytes, le clonage et le séquençage du gène de la tyrosinase ont permis une meilleure compréhension de l'albinisme oculo-cutané tyrosinase négatif (type 1) par la caractérisation d'un nombre important de mutations attachées à cette pathologie [4]. Ces progrès ont également conduit à la description, chez l'homme, d'anomalies pigmentaires dont l'existence n'avait jusqu'alors été constatée que chez l'animal (mutation Himalaya des rongeurs, mutation siamoise du chat). La pigmentation du pelage chez ces animaux est plus marquée sur les extrémités, c'est-à-dire les zones tégumentaires où la température est la plus basse, suggérant une thermosensibilité de la tyrosinase induite par une mutation ponctuelle du gène de cette enzyme. Une situation tout à fait homologue (mutation a été observée chez une famille présentant un albinisme oculo-cutané avec présence de poils blancs axillaires, et de poils pigmentés sur les extrémités [5].

Deux gènes impliqués dans le déterminisme de la structure biochimique des mélanines (eumélanines versus phaeomélanines), les gènes agouti et extension, ont été identifiés chez la souris. Le gène murin agouti a été cloné et séquencé [6], le gène du récepteur de la MSH a été cloné et pourrait être associé au locus extension [7]. Le rôle exact de ces gènes chez l'homme est inconnu.

Plusieurs gènes contrôlant la morphologie et la forme des mélanocytes ont été récemment identifiés. La tyrosinase related proteine I (TRP-1) a été associée au locus brown de la souris. Une mutation dominante de ce locus, appelée light, est caractérisée par la présence de poils pigmentés uniquement à leur extrémité. Cette hypomélanose paraît être le résultat d'une mort prématurée des mélanocytes, peut-être due à la mélanocytotoxicité de certains métabolites intermédiaires de la mélanogenèse. Cette mutation pourrait représenter un modèle animal du grisonnement prématuré des cheveux et/ou du vitiligo humain [8]. Le rôle biologique de la TRP-1 est inconnu. Il semble toutefois que le produit de TRP-1 interagisse avec le produit d'un autre gène murin (silver) qui code pour une protéine de la matrice des mélanosomes.

La démonstration récente que le gène dilute, qui induit une hypomélanose récessive de la souris, code pour un nouveau type de chaîne lourde de la myosine fera certainement progresser notre compréhension des mécanismes moléculaires de la dendritogenèse mélanocytaire et peut-être de la pathogénie de l'hypomélanose associée au syndrome de GriscelliPruniéras [9], une affection induisant une hypomélanose cutanée et des infections fréquentes liées à un déficit immunitaire sévère. L'hypomélanose est corrélée à une anomalie de transfert des mélanosomes aux kératinocytes épidermiques résultant d'une incapacité des mélanocytes à produire des dendrites.
La mutation pink-eyed de la souris a été récemment associée à deux affections comportant une hypomélanose cutanéo-phanérienne, le syndrome de Prader-Willi et le syndrome d'Angelmann [10]. Le produit du gène pinkeyed est inconnu, mais la présence d'anomalies structurales des mélanosomes des souris mutantes suggère qu'il s'agit d'une protéine structurale des mélanosomes. Il a été suggéré que ce gène serait également associé à l'albinisme oculo-cutané tyrosinase positif (type II) et à l'hypomélanose d'Ito (syndrome associant une hypopigmentation cutanée en " jet d'eau " et des anomalies neurologiques : retard mental, ataxie, convulsions...).

La pallidine est la protéine mutée chez les souris pallid qui présentent un allongement du temps de saignement lié à une anomalie plaquettaire, un déficit de sécrétion d'enzymes lysosomiales dans le rein et une dilution de la pigmentation du pelage [11]. Cette protéine (encore appelée protéine 4.2) est vraisemblablement présente dans la membrane des lysosomes, des granules denses des plaquettes et des mélanosomes. L'homologue humain est donc un excellent candidat pour les syndromes d'Hermansky-Pudlak et de ChediakHigashi, qui comportent des altérations des lysosomes, mélanosomes, granules plaquettaires.

Les acquisitions ont été beaucoup moins importantes dans le groupe des hypermélanoses génétiques représentées par les taches café au lait (TCL) et les hypermélanoses dermiques (tache mongolique sacrée ou aberrante, naevus d'Ota). Une mutation du gène de la sous-unité $\alpha$ de la protéine $\mathrm{G}$ activatrice de l'adénylcyclase a été détectée au cours du syndrome de McCune-Albright dont les TCL, segmentaires sont un des éléments symptomatiques habituels [12]. De même, le gène de la neurofibromatose I, associé à de nombreuses TCL, a été cloné et séquencé, et la neurofibromine, produit de ce gène, bien caractérisée. Toutefois, les mécanismes cellulaires et moléculaires déterminant l'apparition des TCL n'ont pas encore été identifiés.

Les hypermélanoses dermiques carac- 
térisées par la présence ectopique de mélanocytes dans le derme sont considérées comme la conséquence d'une anomalie de l'embryogenèse (migration, différenciation des mélanoblastes ?), résultant de mécanismes inconnus. La présence de mélanocytes en situation ectopique (muscle, glandes...) chez des souris transgéniques par l'introduction d'une construction associant l'oncogène ret et le promoter-enhancer de la métallothionéine murine suggère que le récepteur TK codé par ret pourrait jouer un rôle important dans l'embryogenèse du système mélanocytaire et peut-être dans la pathogénie de certaines hypermélanoses ectopiques, comme le naevus d'Ota [13].

Il est prévisible que d'autres gènes de pigmentation vont être bientôt clonés et séquencés. Un travail important va être nécessaire pour définir le rôle de certains des produits des gènes qui ont déjà été clonés. Nul doute que nos connaissances de la pigmentation cutanée et des affections qui lui sont associées vont évoluer extrêmement rapidement

\section{RÉFÉRENCES}

1. Spritz RA, Holmes SA, Ramesar R, Greenberg J, Curtis D, Beighton P. Mutations of the kit (Mast/Stem cell growth factor receptor) proto-oncogene account for a continuous range of phenotypes in human piebaldism. Am J Hum Genet 1992; 51 : 1058-65.

2. Spritz RA, Droetto S, Fukushima Y. Deletion of the kit and PDGFRA genes in a patient with piebaldim. $A m \mathrm{~J}$ Med Gen $1992 ; 44: 492-5$.

3. Tassabehji M, Read AP, Newton VE, Patton M, Gruss P, Harris R, Strachan T. Mutations in the Pax- 3 gene causing Waardenburg syndrome type 1 and type 2. Nature Genet 1993 ; 3 : 26-30.

4. Tripathi RK, Strunk KM, Giebel LB, Weleber RG, Spritz RA. Tyrosinase gene mutations in type I (tyrosinase-deficient) oculocutaneous albinism define two clusters of missense substitutions. Am J Med Gen $1992 ; 43: 865-71$

5. Giebel LB, Tripathi RK, King RA Spritz RA. Tyrosinase gene missense mutation in temperature-sensitive type 1 oculocutaneous albinism. A human homologue to the Siamese cat and the Himalayan mouse. $J$ Clin Invest 1991; $87:$ 1119-22.

6. Bultman SJ, Michaud EJ, Woychik RP. Molecular characterization of the mouse Agouti locus. Cell 1992; 71: 1195-204.

7. Mountjoy KG, Robbins LS, Mortrud MT, Cone RD. The cloning of a family of genes that encode the melanocor- tin receptors. Science $1992 ; 257: 1248-51$. 8. Johnson R, Jackson IJ. Light is a dominant mouse mutation resulting in premature cell death. Nature Genet 1992; 1: 226-9.

9. Mercer JA, Seperack PK, Strobel MC, Copeland NG, Jenkins NA. Novel myosin heavy chain encoded by murine dilute coat colour locus. Nature 1991; 349 : 709-12.

10. Brilliant $\mathrm{MH}$. The mouse pink-eyed dilution locus : a model for aspects of Prader-Willi syndrome, Angelman syndrome, and a form of hypomclanosis of Ito. Mammalian Genome 1992; 3 : 187-91. 11. White RA, Peters LL, Adkison LR, Korsgren C, Cohen CM, Lux SE. The murine pallid mutation is a platelet storage pool disease associated with the protein 4.2 (pallidin) gene. Nature Genet 1992 ; $2: 80-3$.

12. Schwindinger WF, Francomano CA, Levine MA. Identification of a mutation in the gene encoding the $\alpha$ subunit of the stimulatory $\mathrm{G}$ protein of adenylyl cyclase in McCune-Albright syndrome. Proc Natl Acad Sci USA 1992 ; 89 : 5152-6.

13. Iwamoto $T$, Takahashi $\mathbf{M}$, Ito $\mathbf{M}$, Hamatani K, Ohbayashi M, Wajjwalku W, Isobe K, Nakashima I. Aberrant melanogenesis and melanocytic tumour development in transgenic mice that carry a metallothionein/ret fusion gene. EMBO J $1991 ; 10$ : 3167-75.

\section{TIRÉS A PART}

J.-P. Ortonne. 